\title{
Integrating Polarized Light Microscopy with Vibrational Spectroscopy for Solid-State Characterization of Drug Substances
}

\author{
J. A. Reffner, J. A. Seelenbinder, and M. L. Norman \\ Smiths Detection - Danbury, 14 \\ Commerce Drive, Danbury, CT 06810
}

Combining polarized light microscopy with infrared spectroscopy is a unique paring of microanalysis methods for rapid solid-state characterization of active pharmaceutical ingredients (APIs). The API must be analyzed in its solid form, not after it is dissolved or vaporized. X-ray diffraction (XRD), polarized light microscopy (PLM), vibrational spectroscopy (infrared and Raman), and solid-state NMR are useful techniques. Thermal analysis provides additional information on the API's stability and thermodynamic properties. XRD provides information of long range order of crystal lattice while vibrational spectroscopy is sensitive to short range molecular interactions and chemistry. PLM provides crystallographic information and may let you directly observe phase transformations. Each of these methods has its strengths and weaknesses. It is always necessary to know two things, the lattice geometry and the chemistry. Recording changes in lattice geometry is just one-half; knowing the chemistry is the other. While no single method is adequate infrared and Raman spectra can monitor both.

Solid drug substances dominate pharmaceutical research and development. The solid form of an API can be crystalline or amorphous, but crystalline solids are the most common form formulated into drug products. If a crystalline material is chosen, then a polymorph screen is conducted to determine the number and stability of solid-state forms. Most drug substances exist in more than one solid-state form; they are, in the chemical sense, polymorphic. Each polymorphic form of a substance has the identical molecular chemistry, but each form has a unique crystalline lattice. Solubility can affect the bioavailability of the drug. Since solubilities and thermodynamic stability vary between polymorphic forms, it is easy to understand that some polymorphs of an API work better than others. Selecting which solid-state form to develop is a major decision. In addition, the form selection process includes considering a salt or cocrystal. Hydrates, solvates, hydrochlorides, salts, molecular compounds, and complexes may be more efficacious modifications of API's; and each modification may be polymorphic.

The infrared microprobe combines PLM with infrared spectroscopy, and when fitted with a heating, cooling or environmental stage, provides a microanalysis system for the solid-state characterization of APIs. Thermal analysis is valuable part of an API polymorphic screen. Knowing the thermodynamic stability, as well as the relative stability of known forms, aids in development and as important data for stability studies.

Numerous example of the importance of polymorphs in modern drug development are found in the literature [1]. An interesting metric of the polymorphism's importance is the number of U.S. Patents issued that involve polymorphism of drugs, Fig. 1. 
In this work, examples solid-state characterizations of drugs, using infrared microprobe analysis are presented. Carbamazepine (CBZ), in an anti-convulsant used to treat epilepsy. CBZ exists in at least four polymorphic forms as well as a di-hydrate. This drug is a good example for the need to accurately characterize polymorphic form and hydration state since it has been found to lose bioactivity upon absorption of moisture related to the formation of the dihydrate. CBZ was recrystallized from acetone and methanol solutions and by fusion. Each recrystallization process produced different morphology, Fig. 2. The infrared spectra of crystals from each of the recrystallization are shone in Fig. 3. While the crystal habit of CBZ recrystallized from acetone and methanol are quite different their infrared spectra indicate these are the same polymorphic form. However, recrystallizing CBZ by fusion produces a different polymorph. When the solvent's recrystallized material is heated it undergoes a polymorphic transformation at $51^{\circ} \mathrm{C}$. Fig. 4 , reports the changes in the infrared spectra of CBZ as it is heated.

[1] Threlfall, T. L. "Analysis of Organic Polymorphs" Analyst. Vol. 120, 2435-2460 (1995)
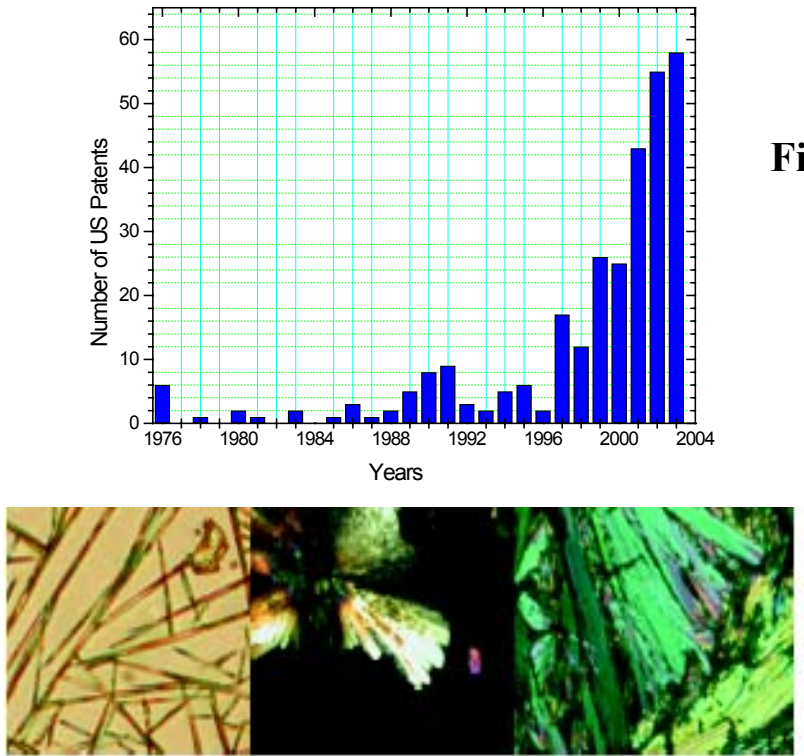

Fig. 3. Infrared spectra of CBZ crystals

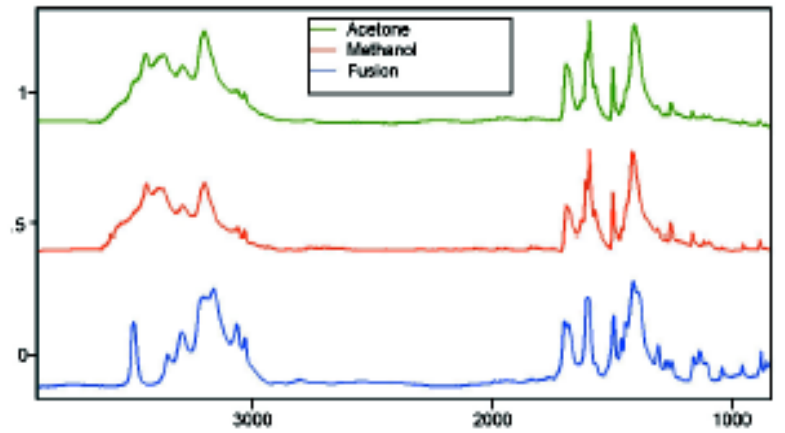

Fig. 1. Number of U.S Patents referring to polymorphic Drugs.

Fig. 2. Recrystallizing of CBZ

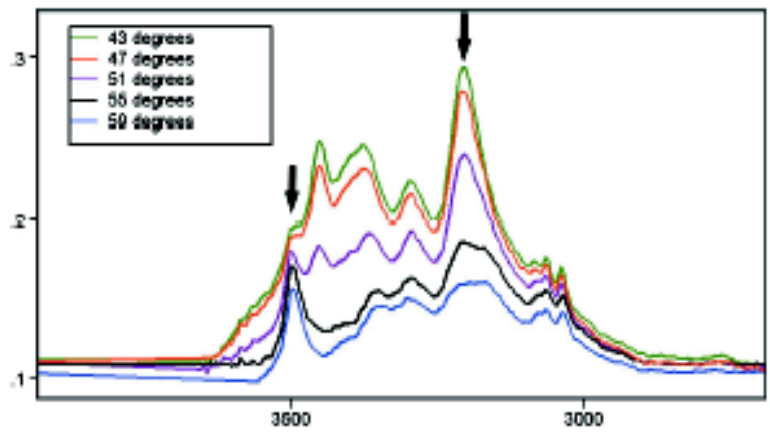

